\title{
Controlling the depth of anesthesia by a novel positive control strategy is
}

\author{
F. N. Nogueira ${ }^{a, b}$, T. Mendonça $a^{c, b}$, P. Rocha ${ }^{a, b}$ \\ ${ }^{a}$ Faculdade de Engenharia da Universidade do Porto, Porto, Portugal. \\ ${ }^{b}$ CIDMA - Center for Research and Development in Mathematics and Applications, \\ Department of Mathematics, University of Aveiro, Portugal. \\ ${ }^{c}$ Faculdade de Ciências da Universidade do Porto, Porto, Portugal.
}

\begin{abstract}
In this paper a positive control law is designed for multi-input positive systems that ensures asymptotic tracking of a desired output reference value. This control law can be viewed as a generalization of another one proposed in the literature for the control of the total mass in SISO compartmental systems, but is suitable for a wider class of positive systems. The controller proposed here is applied to the control of the depth of anesthesia (DoA), by means of the administration of propofol and remifentanil, when using a parameter parsimonious Wiener model recently introduced in the literature. Its performance is illustrated by realistic simulations.

Keywords:

\footnotetext{
This work was supported by FEDER founds through COMPETE-Operational Programme Factors of Competitiveness ("Programa Operacional Factores de Competitividade") and by Portuguese funds through the Center for Research and Development in Mathematics and Applications (University of Aveiro) and the Portuguese Foundation for Science and Technology ("FCT-Fundação para a Ciência e a Tecnologia"), within project PEst-C/MAT/UI4106/2011 with COMPETE number FCOMP-01-0124-FEDER-022690. The authors also acknowledges GALENO - Modeling and Control for personalized drug administration, Funding Agency: FCT,Programme: FCT PTDC/SAU-BEB/103667/2008. Additionally, Filipa Nogueira acknowledges the support of FCT - Portugal through the grant SFRH/BD/48314/2008.
} 
Positive systems, control, DoA, anesthesia.

\section{Introduction}

The goal of automatically controlled drug administration is to determine the dosage to be administered to achieve and keep a certain effect of a drug in the patient. In this work, the primary goal is to track the level of the depth of anesthesia, here measured by the bispectral index (BIS), which is transformed into a problem of tracking the effect concentration by inverting the generalized Hill equation. This problem can be modeled as an output reference tracking problem. More specifically, after modeling the phenomenon in question by a control system in which the control input is the dosage of drug to be administered and the output is the corresponding effect, one seeks a control law that forces the system output to converge to the desired reference value.

Since the quantities involved in this process are all nonnegative, this problem falls within the realm of positive systems. These systems have gained increasing attention in the control literature during the last decades. See for example Farina and Rinaldi [1], Haddad et al. [2], Roszak and Davison [3], Roszak and Davison [4],Kaczorek [5], Willems [6], Soltesz et al. [7]. In this latter reference, a controller by integral action together with a positivity constraint was proposed, showing that the input is positive provided that the integral gain $\epsilon>0$ is chosen sufficiently small. This controller does not presuppose a full knowledge of the model, however it does need the a priori knowledge of the steady-state gain matrix, in order to obtain a suitable value for the integral gain $\epsilon$. This may take a long time to obtain in case the 
process poles are not fast enough. This constitutes a disadvantage for its use in our application. The controller presented in this paper ensures reference tracking independently from the positive value of the design parameter, without requiring the knowledge of the steady-state gain matrix. Nevertheless, it does require information about the model parameters and the corresponding state. However, these parameters can be identified in a short preliminary stage, and a state observer can be included in order to estimate the state online. The control law, developed in this work, is considered to be nonlinear, not due to use of nonlinear design methods, but rather because of the imposition of positivity constraint on the control variable, which makes it a nonlinear function of the state of the system.

In this paper we consider single output positive systems with multiple inputs and design a nonlinear positive control law that ensures asymptotic tracking of a desired output reference value. This control law can be viewed as a generalization of the one proposed in Bastin and Provost [8] for the control of the total mass in SISO compartmental systems. However, whereas the control law in Bastin and Provost [8] is only designed for compartmental systems, our control law is suitable for a wider class of positive systems as is sustained by the new theoretical results presented in the paper (Section 2).

Our results prove to be useful for the control of the depth of anesthesia, a problem that has lately deserved much attention. For instance, the work developed in Soltesz et al. [7] presents two controllers in parallel for the DoA of a patient based on a PID controller for the administration of propofol and a proportional controller for the input of remifentanil. Here, we present one single multi-output feedback controller for both drugs. A good overview of 
the underlying problem may be found in Dumont [9] and in the references there in.

The proposed controller is applied to the control of the depth of anesthesia by means of propofol and remifentanil using the recently proposed parameter parsimonious Wiener model (see Silva et al. [10]). The performance of the controller is analyzed by means of several simulations along with realistic simulations relying on identified real patients data collected in the surgery room during general anesthesia.

The present paper is structured as follows. In Section 2 a control law is designed for output reference tracking in MISO positive systems. The application of the corresponding controller in general anesthesia is presented in Section 3. In Section 4 the performance of the proposed controller is illustrated by means of several simulations, and the results of its application in realistic simulated patients are presented in Section 5. Conclusions follow in Section 6.

\section{Output Reference Tracking for MISO Positive Systems}

In this section the general problem of reference tracking for multi-input/singleoutput (MISO) positive systems is presented. The application to the control of anesthesia will be presented in Section 3.

\subsection{Problem Description}

Consider a positive system with $m$ inputs and a single output described by the state-space model

$$
\left\{\begin{array}{l}
\dot{x}(t)=A x(t)+B u(t) \\
y(t)=C x(t),
\end{array}\right.
$$


where $A$ is a $n \times n$ Meztler matrix, i.e., a matrix in which all the off-diagonal components are nonnegative, and $B$ and $C$ are matrices with nonnegative entries (see Godfrey [11]) of dimension $n \times m$ and $1 \times m$, respectively. Here, for short, in the sequel $(1)$ is denoted by $(A, B, C)$. Moreover, for a vector $v$, the notations $v \geq 0(v>0)$ and $v \leq 0(v<0)$ mean that all its entries are respectively positive (strictly positive) and negative (strictly negative). The same applies to matrices.

Given a desired constant reference value $y^{*}$ for the output, a control law $u=K x+L$ is sought such that the closed-loop system

$$
\left\{\begin{array}{l}
\dot{x}(t)=(A+B K) x(t)+B L \\
y(t)=C x(t)
\end{array}\right.
$$

has bounded trajectories and tracks the reference, i.e., such that its output verifies $\lim _{t \rightarrow \infty} y(t)=y^{*}$.

\subsection{Controller design}

Here we solve the problem of output reference tracking, by regarding it as a problem of controlling the system to a level set $\Omega_{y^{*}}=\left\{x \in \mathbb{R}_{+}^{n}: C x=y^{*}\right\}$ in the state space, where $\mathbb{R}_{+}^{n}=\left\{x \in \mathbb{R}^{n}: x \geq 0\right\}$.

For this purpose, we first design an auxiliary control law, $\tilde{u}$, and then impose positivity to $\tilde{u}$ in order to obtain a positive control input $u$. We also make the following assumptions: $(A 1) A$ is stable, $(A 2) C B$ is a nonzero row matrix and $(A 3) C A<0$.

Let

$$
\tilde{u}(t)=-E C A x(t)+E \lambda\left(y^{*}-y(t)\right)
$$

where $\lambda>0$ is a design parameter, and $E$ is a column matrix with nonnegative entries such that $C B E=1$. Note that such a matrix always exists since 
$C B$ has nonnegative entries, at least one of each is strictly positive. The application of this control input leads to the closed-loop dynamics

$$
\dot{x}(t)=A x(t)+B\left(E \lambda\left(y^{*}-y(t)\right)-E C A x(t)\right)
$$

which implies that

$$
\begin{aligned}
\dot{y}(t) & =C \dot{x}(t)=C A x(t)+\lambda\left(y^{*}-y(t)\right)-C A x(t) \\
& =-\lambda\left(y(t)-y^{*}\right)
\end{aligned}
$$

and therefore

$$
y\left(\hat{\overline{)-}} y^{*}=-\lambda\left(y(t)-y^{*}\right)\right.
$$

Hence, $y(t)-y^{*}=e^{-\lambda t}\left(y(0)-y^{*}\right)$ and

$$
\lim _{t \rightarrow \infty} y(t)=y^{*}
$$

which means that the output reference value is asymptotically tracked.

In the sequel it is shown that reference tracking can still be achieved even when a positivity restriction to the control input is imposed. This positivity restriction is made componentwise and corresponds to taking the control input as $u=\left[\begin{array}{lll}u_{1} & \cdots & u_{m}\end{array}\right]^{T}$ with $u_{i}=\max \left(\tilde{u}_{i}, 0\right)$, where $\tilde{u}_{i}$ denotes the $i-t h$ component of $\tilde{u}$. Note that $\tilde{u}_{i}=E_{i}\left(-C A x+\lambda\left(y^{*}-y\right)\right)$, where $E_{i} \geq 0$ 
is the $i-t h$ entry of $E$. Therefore if $\tilde{u}_{i}<0$ then $-C A x+\lambda\left(y^{*}-y\right)<0$, and all the other components $\tilde{u}_{j}$ of $\tilde{u}$ corresponding to nonzero $E_{j}$ are negative as well. This allows to conclude that either $u=\tilde{u}$ or $u=0$. In this latter case

$$
\lambda\left(y^{*}-y\right)<C A x .
$$

Since, by assumption (A3), $C A<0$ and $x \geq 0$, then $C A x \leq 0$ and (9) implies that $y^{*}-y<0$.

To prove that all trajectories converge to $y^{*}$, we apply the LaSalle's invariance principle (see LaSalle [12], Leine and Wouw [13], Liao et al. [14], Bullo [15]) to the Lyapunov function

$$
V(x)=\frac{1}{2}\left(y^{*}-y\right)^{2}
$$

for the system $(1)$ on $\mathbb{R}_{+}^{n}$.

For $u=\tilde{u}$ :

$$
\begin{aligned}
\dot{V}(x) & =-\left(y^{*}-y\right) \dot{y}=-\left(y^{*}-y\right) C \dot{x} \\
& =-\left(y^{*}-y\right) C\left(A x+B\left[E \lambda\left(y^{*}-y\right)-E C A x\right]\right) \\
& =-\lambda\left(y^{*}-y\right)\left(y^{*}-y\right)=-\lambda\left(y^{*}-y\right)^{2} \leq 0
\end{aligned}
$$

For $u=0$ (which can only happen when $y^{*}-y<0$, see $(3)$ ):

$$
\dot{V}(x)=-\left(y^{*}-y\right) C \dot{x}=-\underbrace{\left(y^{*}-y\right)}_{<0} \underbrace{C A x}_{\leq 0} \leq 0
$$


Thus

$$
\dot{V}(x)= \begin{cases}-\lambda\left(y^{*}-y\right)^{2} & \text { for } u=\tilde{u} \\ -\left(y^{*}-y\right) C A x & \text { for } u=0\end{cases}
$$

By the LaSalle's invariance principle, all system trajectories converge to the largest set contained in

$$
W=\left\{x \in \mathbb{R}_{+}^{n}: \dot{V}(x)=0\right\},
$$

which is forward-invariant under the closed-loop dynamics. It follows from (15) that $\dot{V}(x)=0$ either when $u=\tilde{u}$ and $y=y^{*}$ or when $u=0$,which implies $y^{*}<y$, and $C A x=0$. So we get

$$
W=\left\{x \in \mathbb{R}_{+}^{n}: y=y^{*} \text { or }\left(y^{*}<y \text { and } C A x=0\right)\right\} \text {. }
$$

Moreover, the set $\Omega_{y^{*}}$ of positive states for which the corresponding output $y$ equals $y^{*}$ is forward-invariant under the closed-loop dynamics. In fact, let $F(x)$ be the vector field associated with the closed-loop system

$$
\left\{\begin{aligned}
\dot{x} & =A x+B u \\
u & =\max (\tilde{u}, 0) .
\end{aligned}\right.
$$

When $C x=y^{*}, u=\tilde{u}=-E C A x \geq 0$ and $F(x)=A x-B E C A x$. As a consequence, recalling that $C B E=1$, one obtains $C F(x)=0$ showing that $F(x)$ is tangent to $\Omega_{y^{*}}$. So $\Omega_{y^{*}}$ is forward-invariant under the closed-loop dynamics.

On the other hand, the trajectories starting in a state $x$ for which $C x>y^{*}$ and $C A x=0$ converge to $\Omega_{y^{*}}$, because 


$$
\begin{aligned}
C x>y^{*} & \Rightarrow u=0 \\
& \Rightarrow \dot{x}(t)=A x(t) .
\end{aligned}
$$

Since $A$ is assumed to be stable, if the control would remain zero, then $\lim _{t \rightarrow \infty} C x(t)$ would be zero. This implies that at a certain time instant, say $t^{*}, C x\left(t^{*}\right)$ reaches the value $C x\left(t^{*}\right)=y^{*}$, i.e. $x\left(t^{*}\right) \in \Omega_{y^{*}}$. From this instant on the trajectories remain indefinitely in the forward-invariant set $\Omega_{y^{*}}$. Therefore, the largest invariant subset contained in $W$ is $\Omega_{y^{*}}$ and, by LaSalles's invariance principle, all the closed-loop system trajectories converge to this set, which means that $\lim _{t \rightarrow \infty} y(t)=y^{*}$ as desired.

The study just developed leads to the following result.

Theorem 1. Let $(A, B, C)$ be a positive MISO linear system, such that $A$ is stable, $C A<0$ and $C B \neq 0$. If $u=\max (\tilde{u}, 0), \tilde{u}=-E C A x+E \lambda\left(y^{*}-\right.$ $y)$, with $\lambda>0$, and $E \geq 0$ such that $C B E=1$, then the closed-loop system output $y(t)$ verifies $\lim _{t \rightarrow \infty} y(t)=y^{*}$.

\section{CONTROL OF THE DEPTH OF ANESTHESIA}

Combinations of drugs are used in general anesthesia because no single drug is able to provide all its necessary components (namely, analgesia, hypnosis, areflexia) without seriously compromising hemodynamic and/or respiratory function, impairing operating conditions, or delaying postoperative recovery. Ideal combination of dosing facilitates optimal therapeutic effect without producing significant side effects. 
Here, a control law is designed to administer the hypnotic agent propofol and the opioid analgesic remifentanil to patients during surgery, in order to achieve a desired level of unconsciousness. This is measured in terms of the depth of anesthesia (DoA), usually denoted by $z(t)$, which is a feature that can be related to the quantities of administered drugs as explained next.

In what concerns DoA, the response to the administration of hypnotics and analgesics is commonly modeled as a high order pharmacokinetic/pharmacodynamic (PK/PD) Wiener model (see Bailey and Haddad [16]). However, a new Wiener model (parameter parsimonious Wiener model) with a reduced number of parameters describing the join effect of propofol and of remifentanil as been introduced in Silva et al. [10]. Here, the main goal of this section is to design a controller for the DoA based on this parameter parsimonious Wiener model.

The effect concentration of propofol $\left(c_{e}^{p}\right)$ and of remifentanil $\left(c_{e}^{r}\right)$ can be modeled by the parameter parsimonious Wiener model developed by Silva et al. [10]. According to this model

$$
\begin{aligned}
& c_{e}^{p}(s)=\frac{k_{1} k_{2} k_{3} \alpha^{3}}{\left(k_{1} \alpha+s\right)\left(k_{2} \alpha+s\right)\left(k_{3} \alpha+s\right)} u^{p}(s), \\
& c_{e}^{r}(s)=\frac{l_{1} l_{2} l_{3} \eta^{3}}{\left(l_{1} \eta+s\right)\left(l_{2} \eta+s\right)\left(l_{3} \eta+s\right)} u^{r}(s),
\end{aligned}
$$

where $c_{e}^{p}(s), c_{e}^{r}(s)$ denote the Laplace transforms of $c_{e}^{p}(t)$ and $c_{e}^{r}(t)$, respectively, and $u^{p}(s)$ and $u^{r}(s)$ are the Laplace transforms of the administered doses of propofol, $u^{p}(t)$, and of remifentanil, $u^{r}(t)$, in $m g \mathrm{~min}^{-1}$. Each of 
the transfer functions in (21) and (22) has three aligned poles, more concretely, the first one has poles $\left(-k_{1},-k_{2},-k_{3}\right) \alpha$ and the second one has poles $\left(-l_{1},-l_{2},-l_{3}\right) \eta$. The parameters $k_{j}, l_{j}, j=1,2,3$ were chosen in Silva et al. [10] according to the collected patient data and fixed at the values $k_{1}=10$, $k_{2}=9, k_{3}=1, l_{1}=3, l_{2}=2, l_{3}=1$. The parameters $\alpha$ and $\eta$ are patient dependent.

The joint effect of the concentrations of propofol and remifentanil on the BIS level is modelled in Silva et al. [10] by the generalized Hill equation:

$$
z(t)=\frac{z_{0}}{1+\left(\mu U^{p}+U^{r}\right)^{\gamma}},
$$

where $\mu, \gamma$ are patient dependent parameters, $z_{0}$ is the effect at zero concentration, and $U^{p}$ and $U^{r}$ respectively denote the potencies of propofol and remifentanil, which are obtained by normalizing the effect concentrations with respect to the concentrations that produce half the maximal effect when the drug acts isolated (denoted by $E C_{50}^{p}$ and $E C_{50}^{r}$, respectively), i.e.:

$$
U^{p}=\frac{c_{e}^{p}}{E C_{50}^{p}} \text { and } \quad U^{r}=\frac{c_{e}^{r}}{E C_{50}^{r}}
$$

In this work we consider $\alpha \in[0.03,0.17], \eta \in] 0,5.70], E C_{50}^{p}=10$ and $E C_{50}^{r}=0.01$. The values of $E C_{50}^{p}=10$ and $E C_{50}^{r}=0.01$ are taken from Mendonça et al. [17] and the intervals for the values of $\alpha$ and $\eta$ are obtained as follows: $\alpha \in\left[\bar{\alpha}-2 \sigma_{\alpha}, \bar{\alpha}+4 \sigma_{\alpha}\right]$ and $\left.\left.\beta \in\right] 0, \bar{\beta}+4 \sigma_{\beta}\right]$, where $\bar{\alpha}$ and $\sigma_{\alpha}$ respectively denote the mean and standard deviation of the parameters identified in Mendonça et al. [17], and $\bar{\eta}$ and $\sigma_{\eta}$ have the obvious meaning, 
now for the parameter $\eta$. The choice of lower bound 0 for $\eta$ is due to the fact that $\eta$ should be positive and $\bar{\eta}-2 \sigma_{\eta}<0$.

The transfer functions in (21) and (22) can be represented by the following state-space model:

$$
\left\{\begin{array}{l}
\dot{x}^{i}=A^{i} x^{i}+B^{i} u^{i} \\
c_{e}^{i}=\left[\begin{array}{lll}
0 & 0 & 1
\end{array}\right] x^{i}, \quad i=p, r
\end{array}\right.
$$

where

$$
\begin{aligned}
& x^{i}=\left[\begin{array}{l}
x_{1}^{i} \\
x_{2}^{i} \\
x_{3}^{i}
\end{array}\right] \text { is the state, } \\
& A^{p}=\left[\begin{array}{ccc}
-10 \alpha & 0 & 0 \\
9 \alpha & -9 \alpha & 0 \\
0 & \alpha & -\alpha
\end{array}\right], A^{r}=\left[\begin{array}{ccc}
-3 \eta & 0 & 0 \\
2 \eta & -2 \eta & 0 \\
0 & \eta & -\eta
\end{array}\right], \\
& B^{p}=\left[\begin{array}{c}
10 \alpha \\
0 \\
0
\end{array}\right] \text { and } B^{r}=\left[\begin{array}{c}
3 \eta \\
0 \\
0
\end{array}\right] .
\end{aligned}
$$

Defining $U=\mu U^{p}+U^{r}$ yields

$$
z(t)=\frac{z_{0}}{1+U^{\gamma}}
$$


with

$$
U=\frac{1}{E C_{50}^{p}} \mu c_{e}^{p}+\frac{1}{E C_{50}^{r}} c_{e}^{r}=0.1 \mu c_{e}^{p}+100 c_{e}^{r} .
$$

This leads to the following model

$$
\left\{\begin{array}{l}
\dot{x}(t)=A x(t)+B u(t) \\
U(t)=0.1 \mu c_{e}^{p}(t)+100 c_{e}^{r}(t)=C x(t) \\
z(t)=\frac{z_{0}}{1+U^{\gamma}}
\end{array}\right.
$$

where

$$
\begin{aligned}
& x(t)=\left[\begin{array}{l}
x^{p}(t) \\
x^{r}(t)
\end{array}\right], \quad A=\left[\begin{array}{cc}
A^{p} & 0_{3 \times 3} \\
0_{3 \times 3} & A^{r}
\end{array}\right], \\
& B=\left[\begin{array}{cc}
B^{p} & 0_{3 \times 1} \\
0_{3 \times 1} & B^{r}
\end{array}\right], \quad C=\left[\begin{array}{llllll}
0 & 0 & 0.1 \mu & 0 & 0 & 100
\end{array}\right] .
\end{aligned}
$$

As mentioned before, for surgery purposes it is desirable to maintain the BIS close to a certain reference level $z^{\text {ref }}$ between 40 and 60 . This can be achieved by designing a control law that forces $U(t)$ to follow the constant reference level

$$
U^{r e f}=\sqrt[r]{\frac{z_{0}}{z^{r e f}}-1}
$$

In order to apply the design method of the previous subsection, the product $C B$ should be a nonzero row. However, here $C B=\left[\begin{array}{ll}0 & 0\end{array}\right]$ and the 
condition is not met. To overcome this problem, instead of the output $U(t)$, the output

$$
M(x(t))=0.1 M^{p}\left(x^{p}(t)\right)+100 M^{r}\left(x^{r}(t)\right)=C_{M} x(t)
$$

with

$$
\begin{gathered}
M^{p}\left(x^{p}\right)=\left[\begin{array}{lll}
1 & 1 & 1
\end{array}\right] x^{p}, \quad M^{r}\left(x^{r}\right)=\left[\begin{array}{lll}
1 & 1 & 1
\end{array}\right] x^{r} \text { and } \\
C_{M}=\left[\begin{array}{llllll}
0.1 & 0.1 & 0.1 & 100 & 100 & 100
\end{array}\right],
\end{gathered}
$$

is considered. The notations $M(x(t)), M^{p}\left(x^{p}(t)\right)$, and $M^{r}\left(x^{r}(t)\right)$ are inspired by the fact that, in compartmental systems, the sum of the state components usually corresponds to the total substance mass present in the system. As we shall later see, a connection between the reference value $U^{r e f}$ and an adequate reference value $M^{r e f}$ for $M$ can be established, in such a way that when $\lim _{t \rightarrow \infty} M(x(t))=M^{r e f}$ then $\lim _{t \rightarrow \infty} U(t)=U^{r e f}$ and $\lim _{t \rightarrow \infty} z(t)=z^{r e f}$, as desired.

In a first stage we show that every positive constant reference value $M^{*}$ for $M(x(t))$ can be tracked using a control law as proposed in Theorem 1 .

Then, in a second step, we determine which value should be taken for $M^{*}$ in order to ensure that the desired constant reference value $z^{\text {ref }}$ for the BIS level is achieved.

Since now, for the new output matrix $C_{M}$, defined in (33), $C_{M} B=$ $\left[\begin{array}{ll}\alpha & 300 \eta\end{array}\right]$ is nonzero, the applicability of the proposed controller design method is guaranteed. 
Proposition 1. Let $\left(A, B, C_{M}\right)$ be a positive MISO linear system, with $A$, $B$ as in (30) and $C_{M}$ as in (33). Define

$$
E=\left[\begin{array}{l}
\rho \\
1
\end{array}\right] \frac{1}{\alpha \rho+300 \eta}
$$

where $\rho>0$ is an arbitrary nonnegative value. Then, applying the control law

$$
u=\max (0, \tilde{u})
$$

with

$$
\begin{aligned}
\tilde{u}=\left[\begin{array}{c}
\tilde{u}^{p} \\
\tilde{u}^{r}
\end{array}\right] & =E\left(-C_{M} A x+\lambda\left(M^{*}-M\right)\right) \text { and } \lambda>0 \\
& =\left[\begin{array}{c}
\rho \\
1
\end{array}\right] \underbrace{\left(-C_{M} A x+\lambda\left(M^{*}-M\right)\right) \frac{1}{\alpha \rho+300 \eta}}_{\bar{u}}, \\
& \text { and } \lambda>0
\end{aligned}
$$

to the system $\left(A, B, C_{M}\right)$, yields $\lim _{t \rightarrow \infty} M(x(t))=M^{*}$.

Proof. Since $C_{M} B E=1$, all the conditions of Theorem 1 are satisfied yielding the desired result.

Remark: Note that, in this control law, $\lambda$ and $\rho$ are design parameters. Moreover $\left[\begin{array}{l}\tilde{u}^{p} \\ \tilde{u}^{r}\end{array}\right]=\left[\begin{array}{l}\rho \\ 1\end{array}\right] \bar{u}$, with $\bar{u}$ as in (37), meaning that the proportion of propofol and remifentanil is $\rho: 1$.

Since the choice of the positive parameter $\rho$ does not affect the tracked reference value, this may constitute an advantage, as it allows to choose 
the proportion between the two drugs, in order to accommodate clinical restrictions or considerations, without significant consequences in terms of the effect. This will be illustrated later on in the simulations.

In order to determine which value of $M^{\text {ref }}$ should be chosen for $M^{*}$ in the control law (37) to guarantee that the BIS level $z(t)$ tracks a desired constant value $z^{*}$, an analysis is made of the values $U(t)$ obtained for the closed-loop system. For this purpose it will be first proved that the state of the closed-loop system $\left(A, B, C_{M}\right)$ with the control law (35) converges to an equilibrium point $x^{*}$. To show this, since, as mentioned in the previous section, the trajectories $x(t)$ converge to the forward-invariant set $\Omega_{M^{*}}=$ $\left\{x \in \mathbb{R}_{+}^{6}: M(x)=M^{*}\right\}$, it is enough to prove that, for the restriction of the closed-loop system to this set, the state trajectories converge to an equilibrium point $x^{*}$. Note that this restriction is well defined as $\Omega_{M^{*}}$ is forward-invariant under the closed-loop dynamics.

In order to obtain the dynamics of the system restricted to $\Omega_{M^{*}}$, note that when $x \in \Omega_{M^{*}}$, i.e., when $M(x)=M^{*}$, the control $\tilde{u}$ is given by $\tilde{u}=-E C A x$, and the closed-loop system can be described as

$$
\dot{x}=\tilde{A} x
$$

with 


$$
\tilde{A}=A-B E C_{M} A
$$

$$
=\left[\begin{array}{cccccc}
a_{1}-10 \alpha & 8 a_{1} & a_{1} & a_{2} & a_{2} & a_{2} \\
9 \alpha & -9 \alpha & 0 & 0 & 0 & 0 \\
0 & \alpha & -\alpha & 0 & 0 & 0 \\
a_{3} & 8 a_{3} & a_{3} & a_{4}-3 \eta & a_{4} & a_{4} \\
0 & 0 & 0 & 2 \eta & -2 \eta & 0 \\
0 & 0 & 0 & 0 & \eta & -\eta
\end{array}\right]
$$

where

$$
\begin{aligned}
a_{1}= & \alpha^{2} \rho e, \quad a_{2}=1000 \alpha \eta \rho e \\
a_{3}= & 0.3 \alpha \eta e, \quad a_{4}=300 \eta^{2} e \\
e= & \frac{1}{\alpha \rho+300 \eta} \\
& \text { and } A, B, C_{M} \text { and } E \text { are given by (30),(33), and (34). }
\end{aligned}
$$

In order to analyze the stability of the closed-loop system restricted to $\Omega_{M^{*}}$, the evolution equations of this restriction are next obtained.

When $M(x(t))=M^{*}$,

$$
x_{1}^{p}(t)=M^{*}-x_{2}^{p}(t)-x_{3}^{p}(t)-1000\left(x_{1}^{r}(t)+x_{2}^{r}(t)+x_{3}^{r}(t)\right) .
$$

Replacing this in the equation and $\dot{x}(t)=\tilde{A} x(t)$ yields 


$$
\left\{\begin{aligned}
\dot{x}_{1}^{p}(t)= & M^{*}-\dot{x}_{2}^{p}(t)-\dot{x}_{3}^{p}(t)-\dot{x_{1}^{r}}(t)-\dot{x}_{2}^{r}(t)-\dot{x}_{3}^{r}(t) \\
\dot{x_{2}^{p}}(t)= & 9 \alpha\left(M^{*}-x_{2}^{p}(t)-x_{3}^{p}(t)-1000\left(x_{1}^{r}(t)+x_{2}^{r}(t)+x_{3}^{r}(t)\right)\right)-9 \alpha x_{2}^{p}(t) \\
\dot{x_{3}^{p}}(t)= & \alpha x_{2}^{p}(t)-\alpha x_{3}^{p}(t) \\
\dot{x_{1}^{r}}(t)= & a_{3} M^{*}+7 a_{3} x_{2}^{p}(t)+\left(a_{4}-1000 a_{3}-3 \eta\right) x_{1}^{r}(t)+ \\
& +\left(a_{4}-1000 a_{3}\left(x_{2}^{r}(t)+x_{3}^{r}(t)\right)\right. \\
\dot{x}_{2}^{r}(t)= & 2 \eta x_{1}^{r}(t)-2 \eta x_{2}^{r}(t) \\
\dot{x_{3}^{r}}(t)= & \eta x_{2}^{r}(t)-\eta x_{3}^{r}(t) .
\end{aligned}\right.
$$

Since the state component $x_{1}^{p}(t)$ can be obtained from the others state components (see (39)), we can conclude that the closed-loop system dynamics restricted to $\Omega_{M^{*}}$ is described by the evolution of the vector

$$
\bar{x}=\left[\begin{array}{c}
x_{2}^{p} \\
x_{3}^{p} \\
x_{1}^{r} \\
x_{2}^{r} \\
x_{3}^{r}
\end{array}\right],
$$

which is given by the last five equations from (40). These equations can be written in matrix form as:

$$
\dot{\bar{x}}=\bar{A} \bar{x}+R,
$$


with

$$
\bar{A}=\left[\begin{array}{ccccc}
-18 \alpha & -9 \alpha & -9000 \alpha & -9000 \alpha & -9000 \alpha \\
\alpha & -\alpha & 0 & 0 & 0 \\
7 a_{3} & 0 & a_{4}-1000 a_{3}-3 \eta & a_{4}-1000 a_{3} & a_{4}-1000 a_{3} \\
0 & 0 & 2 \eta & -2 \eta & 0 \\
0 & 0 & 0 & \eta & -\eta
\end{array}\right]
$$

and

$$
R=\left[\begin{array}{c}
9 \alpha M^{*} \\
0 \\
a_{3} M^{*} \\
0 \\
0
\end{array}\right]
$$

Moreover, equation (42) can be written in the form:

$$
\overline{\hat{x}} \bar{x}^{*}=\bar{A}\left(\bar{x}-\bar{x}^{*}\right)
$$

where

$$
\bar{x}^{*}=\left[\begin{array}{c}
\rho \\
\rho \\
1 \\
1 \\
1
\end{array}\right] \frac{M^{*}}{3(0.1 \rho+100)}
$$

is the only equilibrium point of (42), i.e., the only point such that $\bar{x}(t) \equiv \bar{x}^{*}$ satisfies $\dot{\bar{x}}=\bar{A} \bar{x}(t)+R$, as $\dot{\bar{x}}(t)=0$ and $\bar{A} \bar{x}^{*}=-R$. This can be verified by performing the corresponding multiplication $\bar{A} \bar{x}^{*}$ and checking that the result 
equals $-R$. Moreover, the uniqueness of $\bar{x}^{*}$ follows from the invertibility of $\bar{A}$ for the considered parameter range.

As is shown in Fig. 1, where the locations of the eigenvalues of $\bar{A}$ are plotted for the different value combinations of the parameters $\alpha$ and $\eta$, within the ranges in this paper, and for $\rho \in[0,500]$, the matrix $\bar{A}$ is stable, i.e., all its eigenvalues have negative real part. The restriction $\rho \in[0,500]$ is only imposed to limit the high computation level, however this feat is not a limitation to the applicability of the controller here proposed, since the $\rho$ values observed in the collected clinical cases are included in the considered interval. Thus, $\bar{x}(t)$ converges to $\bar{x}^{*}=(\rho, \rho, 1,1,1) \frac{M^{*}}{3(0.1 \rho+100)}$. Recalling that equation (39) holds, this implies that $x(t)=\left(x_{1}^{p *}, \bar{x}^{*}\right)$ with

$$
\begin{aligned}
x_{1}^{p *} & =M^{*}-\left[\begin{array}{lllll}
1 & 1 & 100 & 100 & 100
\end{array}\right] \bar{x}^{*} \\
& =\frac{\rho M^{*}}{3(0.1 \rho+100)} .
\end{aligned}
$$

Thus $x(t)$ converges to $x^{*}=(\rho, \rho, \rho, 1,1,1) \frac{M^{*}}{3(0.1 \rho+100)}$ under the closedloop dynamics. 


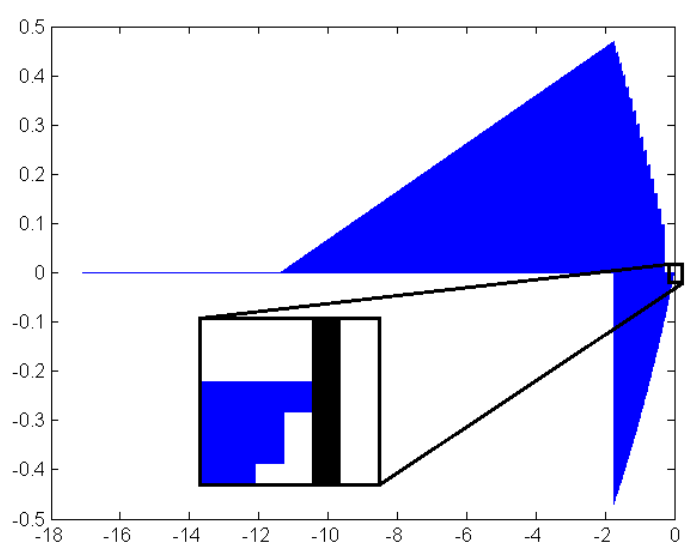

Figure 1: Location of the eigenvalues of the matrix $\bar{A}$, for the different value combinations of the parameters $\alpha, \eta$ and $\rho$ within the ranges in this paper, i.e., $\alpha \in[0.03,0.17]$, $\eta \in] 0,5.70], \rho \in[0,500]$

Recalling that $u(t)=0.1 \mu x_{3}^{p}(t)+100 x_{3}^{r}(t)$, one concludes that, under the closed loop dynamics,

$$
\begin{aligned}
U^{*}=\lim _{t \rightarrow \infty} U(t) & =0.1 \mu \lim _{t \rightarrow \infty} x_{3}^{p}(t)+100 \lim _{t \rightarrow \infty} x_{3}^{r}(t) \\
& =0.1 \mu \frac{\rho M^{*}}{3(0.1 \rho+100)}+100 \frac{M^{*}}{3(0.1 \rho+100)} \\
& =M^{*} \frac{0.1 \mu \rho+100}{3(0.1 \rho+100)} .
\end{aligned}
$$

Consequently, in order to track a constant reference level $U^{r e f}$ for $U(t)$ it is enough to take

$$
M^{*}=M^{r e f}=\frac{3(0.1 \rho+100)}{0.1 \mu \rho+100} U^{r e f}
$$


in the control law (35).

Together with expression (31), these considerations lead to the following result.

Proposition 2. Let $\left(A, B, C_{M}\right)$ be a positive MISO linear system, with $A$, $B$ as in (30) and $C_{M}$ as in (33). Then, applying the control law $u$ as in (35), with $M^{*}=M^{\text {ref }}=\frac{3(0.1 \rho+100)}{0.1 \mu \rho+100} \sqrt[\gamma]{\frac{z_{0}}{z^{r e f}}-1}$, to the system $\left(A, B, C_{M}\right)$, achieves tracking of the constant reference value $z^{\text {ref }}$ for the BIS level $z(t)$ defined as in (23), i.e., $\lim _{t \rightarrow \infty} z(t)=z^{\text {ref }}$.

Proof. It follows from what has just been said that taking in (35)

$$
M^{*}=M^{r e f}=\frac{3(0.1 \rho+100)}{0.1 \mu \rho+100} \sqrt[\gamma]{\frac{z_{0}}{z^{r e f}}-1}
$$

achieves tracking of the constant reference value for $U$ :

$$
U^{r e f}=\sqrt[r]{\frac{z_{0}}{z^{r e f}}-1}
$$

The result now follows from expression (31).

As stated in Proposition 2, once the values of $\gamma$ and $\mu$ are known for a particular patient, the value of $M^{r e f}$ to be considered in the control law is:

$$
M^{r e f}=\frac{3(0.1 \rho+100)}{0.1 \mu \rho+100} \sqrt[r]{\frac{z_{0}}{z^{r e f}}-1}
$$

where $z_{0}=97.7$ and $z^{\text {ref }}$ is the desired BIS level. Here it is assumed that the 
values of $\gamma$ and $\mu$ are available, however, if this is not the case, these values can be identified in a first stage, before turning on the controller. Another possibility is to start with an average value for $\gamma$ and $\mu$ and hence $M^{*}$ as a first estimate and then retune this value after $\gamma$ and $\mu$ are more accurately identified. A similar procedure was used in Nogueira et al. [18]. Here, it is also assumed that the values of the parameters $\alpha, \eta$ are known. This does not happen in practice. However, in a real situation the controller action only starts after an initial drug bolus is given. This is a current practice with other automatic controllers previously used in clinical environment and allows to identify the parameters for patient model.

\section{CONTROLLER PERFORMANCE}

In this section the performance of the control law presented in the previous sections is illustrated by means of several simulations. For this purpose, we consider: $z_{0}=97.7 ; \alpha=0.0759 ; \eta=0.583 ; \mu=1.79 ; \gamma=1.88$. These parameter values are the mean values used in the work developed in Mendonça et al. [17], to which we refer for further explanation. We also assume that it is intended that the DoA of a patient, corresponds to the reference value of 50 for the BIS signal. By (31), this means that $U$ must follow the reference $U^{*}=0.9753$. Once these values are fixed, the control law only depends on the design parameters $\lambda>0$ and $\rho \geq 0$. The parameter $\lambda$ influences the speed of convergence to the reference value, as can be seen in Fig. 2 where the values $\lambda=0.02, \lambda=1$ were respectively taken for a fixed value of $\rho=2$ (corresponding to proportion of $2: 1$ for propofol and remifentanil). 


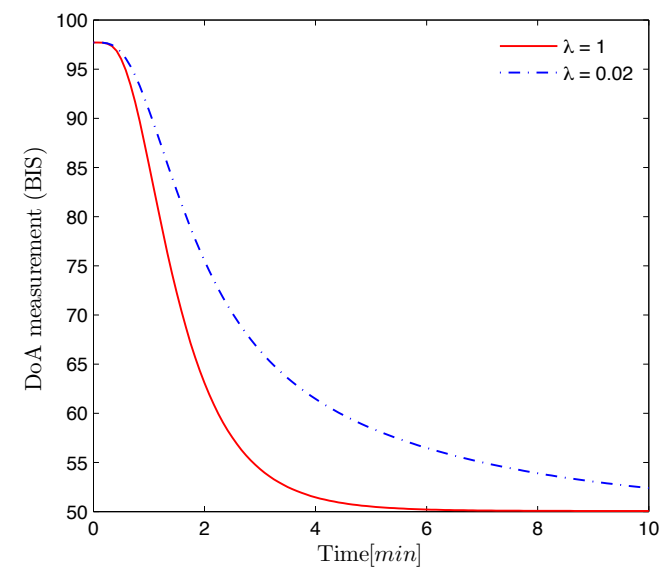

Figure 2: Evolution of DoA, for $\rho=2, \lambda=0.02$ and $\lambda=1$.

On the other hand the parameter $\rho$ specifies the desired proportion between the administered amounts of propofol and remifentanil, which may be chosen according to clinical criteria. Figure 3 illustrates DoA effect for different drug proportions (namely, $\rho=0, \rho=2$, and $\rho=10$ ) and Figures 4,5 , and 6 present the evolution of the corresponding drug dosages. As can be seen in Fig. 3, in spite of the variation of the drug proportion $(\rho)$ the desired effect is practically the same. It turns out that this may constitute an advantage, since the choice of the proportion can be made in order to accommodate individual clinical restrictions or considerations, without significantly consequences in terms of the effect. 


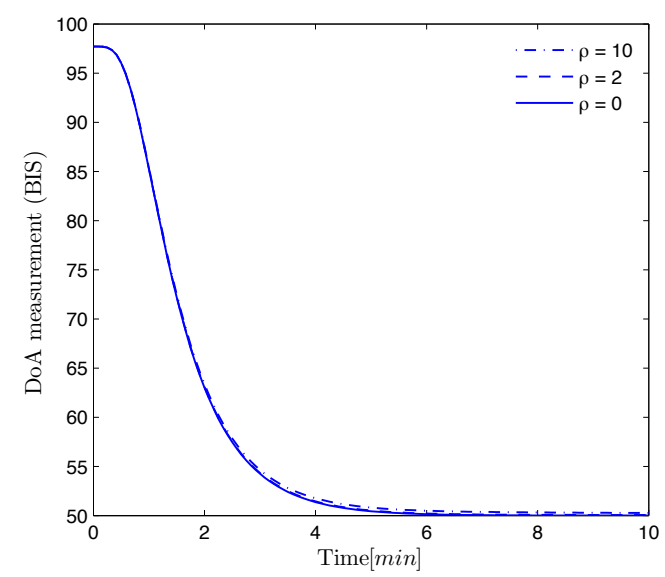

Figure 3: Evolution of DoA for different values of drugs proportion, i.e., $\rho=0, \rho=2, \rho=$ 10 and for $\lambda=1$.

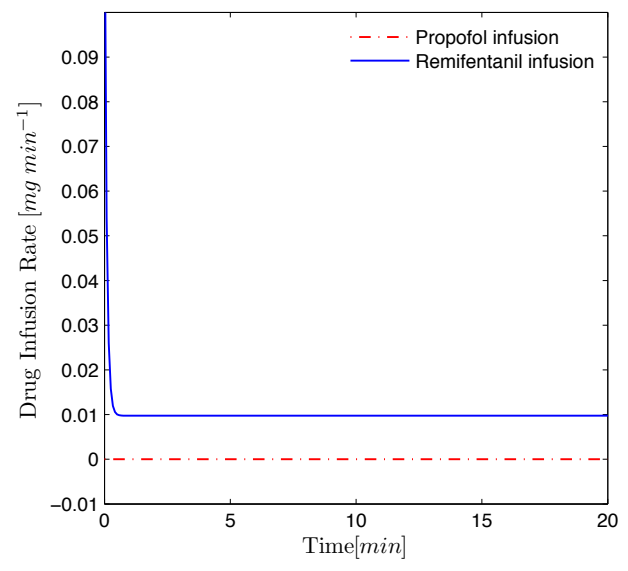

Figure 4: Evolution of the dosage of propofol and of remifentanil, for $\lambda=1, \rho=0$ (without propofol infusion dose). 


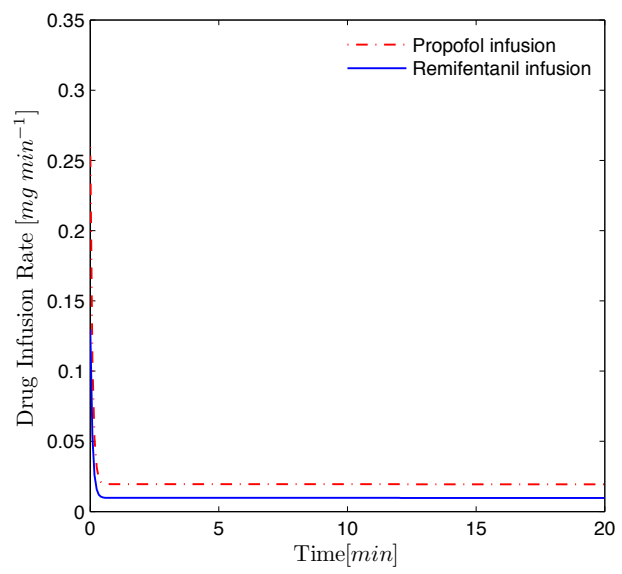

Figure 5: Evolution of the dosage of propofol and of remifentanil, for $\lambda=1, \rho=2$.

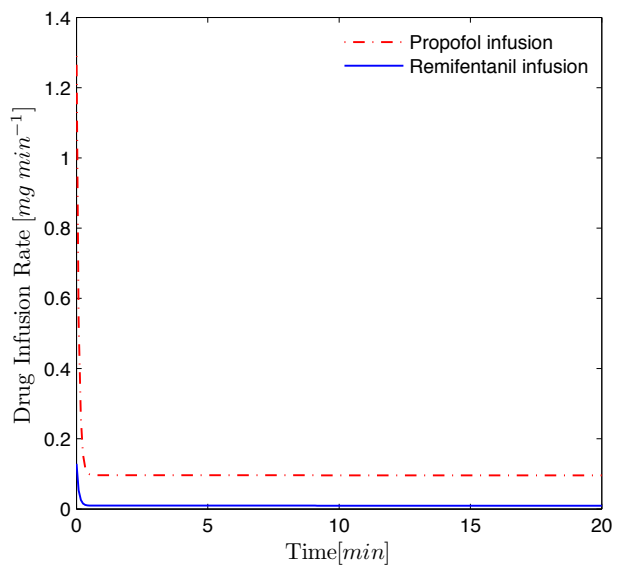

Figure 6: Evolution of the dosage of propofol and of remifentanil, for $\lambda=1, \rho=10$.

Figure 7 illustrates the performance of the control algorithm in the presence of a change of the reference profile. In the first thirteen minutes it is intended that the BIS follows the reference 50, in the following thirteen 
minutes the BIS reference level is set to 30 and in the last twenty minutes the BIS should again follow the reference level 50. It may be seen that the controller has a good performance also in this case. As expected, when the reference decreases there is a small bolus of propofol and remifentanil, in order to follow the reference, and when it increases no drug is administered.
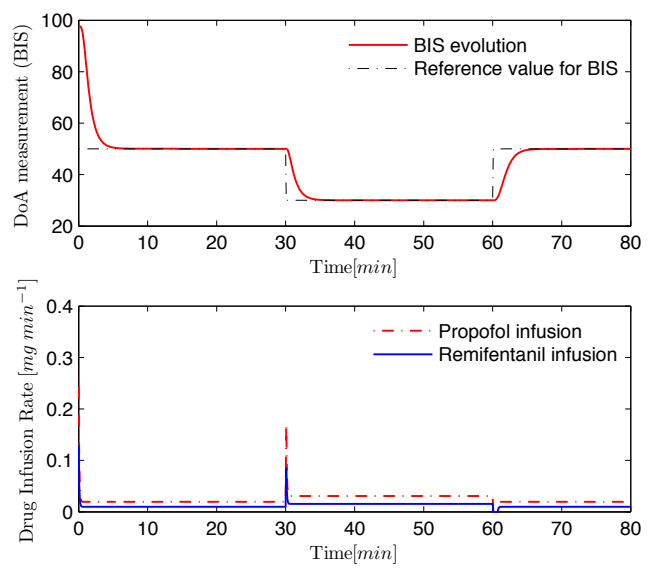

Figure 7: Evolution of DoA and of the dosages of propofol and of remifentanil, assuming changes in the reference profiles $\left(z^{*}=50\right.$ from the beginning till $t=30 \mathrm{~min}, z^{*}=30$ from $t=30 \mathrm{~min}$ till $t=60 \mathrm{~min}$, and $z^{*}=50$ from then on), for $\lambda=1, \rho=2$.

In a real clinical environment, the measurements of the patients BIS levels usually present a high level of noise, due to the nature of the sensors and the physiological ineheretic signs. This fact implies the existence of noise in the estimation of the states in the corresponding theoretical model. In Fig. (8) the evolution of the DoA of a patient is illustrated in the presence of noise in the measurement of the BIS level, that mimics the real data collected in the surgery room. For this purpose, a normal distribution $N\left(\mu_{\text {noise }}, \sigma_{\text {noise }}\right)$, 
where $\mu_{\text {noise }}=0$ and $\sigma_{\text {noise }}=3$ are respectively the mean and the standard deviation, was used. As we can see, in spite of the presence of noise in the BIS measurements, the behavior of the controlled output of the patient is clinically accepted.

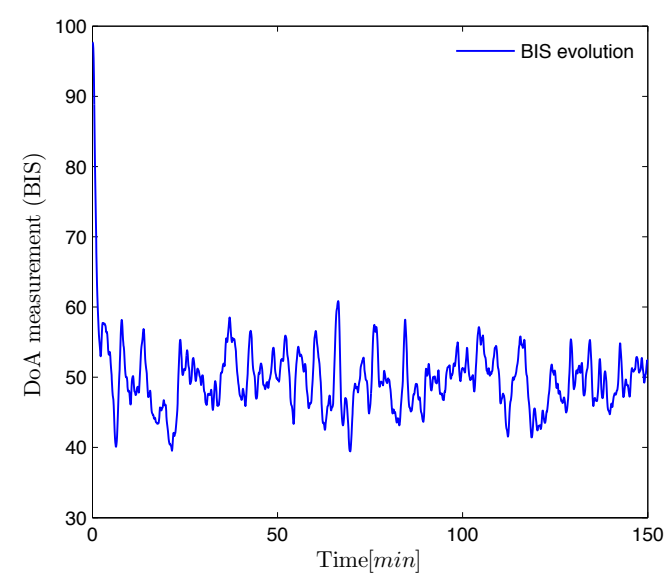

Figure 8: Evolution of DoA in the presence of noise, for $\rho=2$ and $\lambda=1$.

\section{A realistic simulation study}

As a necessary preliminary step before implementation in clinical environment, the performance of the controller proposed in this paper is tested on a bank of realistic simulated patients. These patients are simulated by means of PK/PD Wiener models (see Marsh et al. [19], and Minto et al. [20]) obtained from real data collected during eighteen breast surgeries. The patients (all female, with $54 \pm 13$ years of age, a height of $160 \pm 5 \mathrm{~cm}$, and $69 \pm 18 \mathrm{Kg}$ ) were subject to general anesthesia under propofol and remifentanil administration. The DoA was monitored by the BIS and was manually controlled 
around clinically accepted values by the anesthetist. Alaris GH pumps were used for both propofol and remifentanil. Infusion rates, BIS values and other physiological variables were acquired every five seconds (Mendonça et al. $[17])$.

For each patient, the corresponding PK/PD Wiener model was obtained as follows. The parameters of the linear part were computed according to Marsh et al. [19], Minto et al. [20], and Schnider et al. [21] based on the relevant patient characteristics, whereas the parameters of the nonlinear part (generalized Hill equation) were identified from the surgery data, by Mendonça et al. [17]. In order to test the proposed control strategy, each simulated patient is also modeled by the parsimonious parameter Wiener model of Silva et al. [10], with parameters identified as in Mendonça et al. [17], and a control law of the form (37) tuned for that model is applied to the simulated patient. The set of parameter values identified by Mendonça et al. [17] is given in appendix A, where also the corresponding relevant patient features that allow to compute the PK/PD model parameters are displayed.

Here we present the results obtained for patients 9 and 15 of the database in two different simulations settings respectively. Patient 9 is a woman, with 51 years of age, a height of $163 \mathrm{~cm}$, and with $55 \mathrm{~kg}$. The corresponding identified parameters were: $E C_{50}^{p}=12.17, E C_{50}^{r}=0.031, \gamma=1.86, \mu=3.84$, $\alpha=0.07$, and $\eta=0.28$. Patient 15 is a woman, with 73 years of age, a height of $160 \mathrm{~cm}$, a weight of $75 \mathrm{~kg}$, for which the identified parameters were: $E C_{50}^{p}=12.41, E C_{50}^{r}=0.016, \gamma=1.68, \mu=3.47, \alpha=0.10$, and $\eta=0.04$.

Figure 9 shows the BIS response of the patient 15 to the drug doses administered by the anesthetist during the surgery, along with the response 
of the corresponding PK/PD model to the same drug input. The similarity between the two responses supports the idea of using the identified PK/PD models in order to simulate real patients.
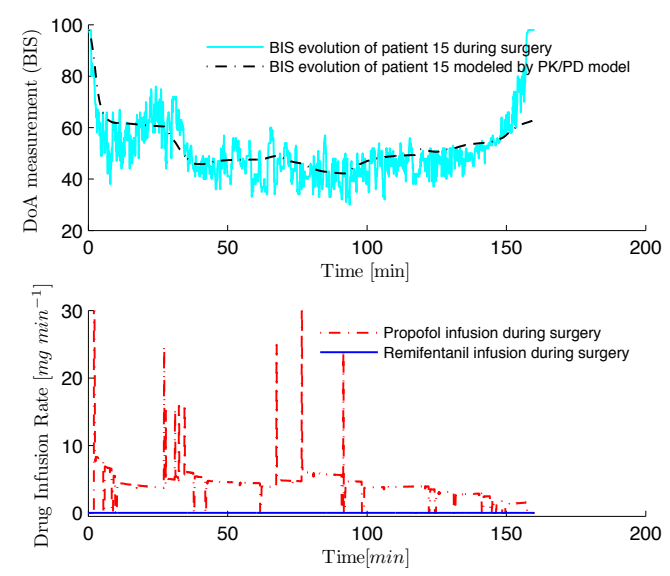

Figure 9: BIS response of the patient 15 (upper plot) to the drug doses administered by the anesthetist during the surgery (lower plot), along with the response of the corresponding PK/PD model to the same drug input. The average doses of propofol and of remifentanil reported were $4.21 \mathrm{mg} \mathrm{min}^{-1}$ and $0.0147 \mathrm{mg} \mathrm{min}^{-1}$ respectively. Notice that propofol doses above $30 \mathrm{mg} \mathrm{min}^{-1}$ are not represented in the graphic.

In Fig. 10 the BIS response of the simulated patient 15 is presented, where the DoA control was performed using the control law (37) for tracking a BIS level of 50; this is an approximation value of the average real BIS obtained throughout the surgery. The selected proportion between the two drugs was the corresponding average reported in the monitored real case, $\rho=286$. The average of the doses of propofol and of remifentanil obtained by the controller were respectively $4.27 \mathrm{mg} \mathrm{min}^{-1}$ and $0.0149 \mathrm{mg} \mathrm{min}^{-1}$; these values are quite close to the reported real ones, $4.21 \mathrm{mg} \mathrm{min}^{-1}$ and $0.0147 \mathrm{mg} \mathrm{min}^{-1}$ 
respectively. The obtained set-point for the BIS level was 54.7, presenting an relative error of only $9 \%$.

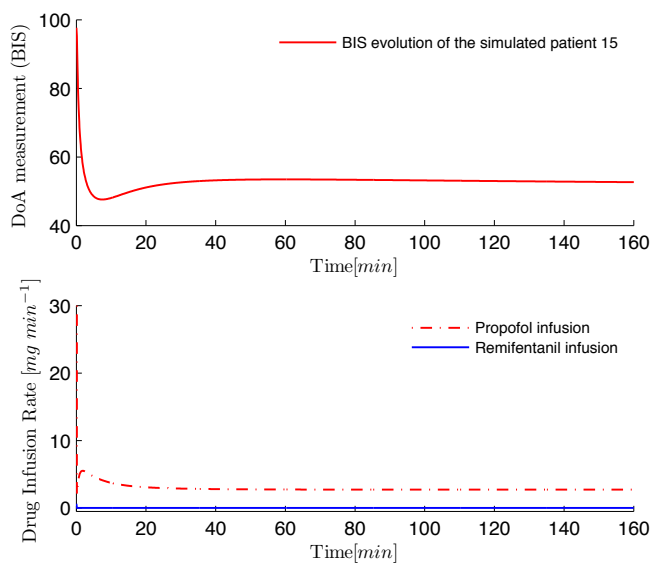

Figure 10: BIS response of the patient 15 and the infusion doses of propofol and of remifentanil obtained by the proposed control law, for $z^{*}=50, \rho=286$, and $\lambda=10$. The average doses of propofol and of remifentanil obtained by the controller were respectively $4.27 \mathrm{mg} \mathrm{min}^{-1}$ and $0.0149 \mathrm{mg} \mathrm{min}^{-1}$. Notice that propofol doses above $30 \mathrm{mg} \mathrm{min}^{-1}$ are not represented in the graphic.

Figure 11 illustrates the application of the proposed control law to all the 18 simulated patients, for a desired reference BIS level of 50 (the remaining parameters are presented in the figure). As can be seen, the desired BIS level obtained in all the cases are very close to the desired one. 


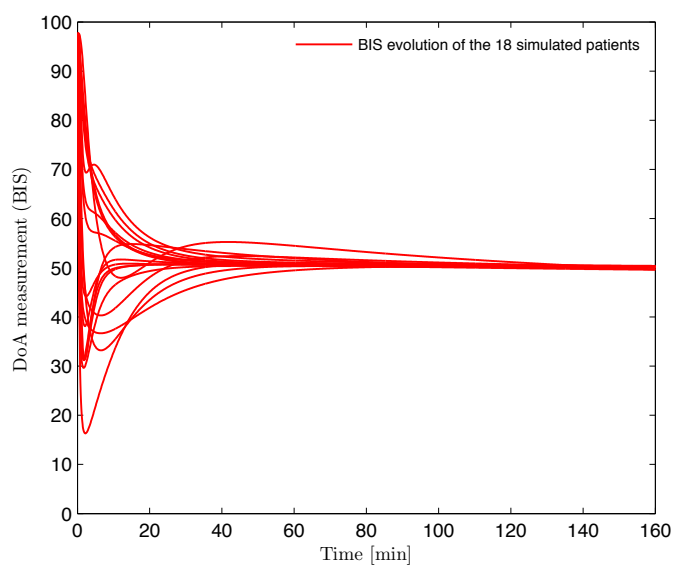

Figure 11: BIS response of all eighteen simulated patients (by means of a PK/PD model) controlled by the proposed control law, tuned for the corresponding parameter parsimonious model, for $z^{*}=50, \rho=2$, and $\lambda=10$.

In Fig. 12 the performance of the control in the presence of a change of the reference profile and in the value of drugs proportion is illustrated (for patient 9). In the first ninety minutes it is intended that the BIS follows the reference 50, in the following thirty minutes the BIS reference level is set to 40 and in the last forty minutes the BIS is regulated to follow again the reference level 50. As expected, a reference decrease produces a peak in the doses of propofol and remifentanil, whereas when the reference increases no drug is administered. The proportion between the two drugs was $\rho=0.2$ in the first forty minutes and from then on it was changed to $\rho=3$. In spite of the variation of the drug proportion the BIS follows the desired reference. The possibility of changing the drug proportion during the surgery without affecting the reference tracking results constitutes a considerable advantage. 
Indeed, clinical practice has often shown the need of adjusting the dosage profile according to the overall physiological response of the patient.

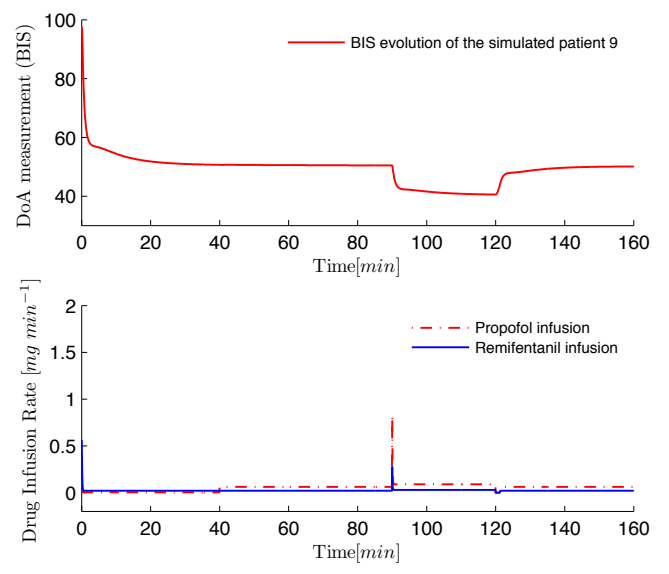

Figure 12: Evolution of DoA, of patient 9, and of the dosages of propofol and of remifentanil, assuming changes in the reference profiles $\left(z^{*}=50\right.$ from the beginning till $t=90 \mathrm{~min}$, $z^{*}=40$ from $t=90 \mathrm{~min}$ till $t=120 \mathrm{~min}$, and $z^{*}=50$ from then on) and assuming changes in the value of the drugs proportions $(\rho=0.2$ in the first forty minutes and from then on it was changed to $\rho=3) . \lambda=1$.

\section{Conclusion}

A positive control law for multi-input positive systems was proposed in order to track a desired output reference value. This controller was applied in the control of the DoA, tracking the BIS level for a certain designed profile, by means of simultaneous administration of propofol and remifentanil, based on the parsimonious parameter model. This new Wiener model describes the joint effect of these two drugs and is adequate for model based control design since it presents a reduced number of parameters. 
In this work it was theoretically proved that the controller may be tuned in order to achieve different convergence rates and different desired proportions between the dosages of these two drugs, in order to obtain a certain reference value for the BIS. Moreover, this controller also provides the possibility of changing the drugs proportion during the surgical anesthetic procedure without affecting the reference tracking results, which constitutes an interesting clinical advantage. Indeed anesthetic procedures during surgery relies on a defined DoA profile, but the change of the dosage profile due to the overall physiological response of the patient is often required.

The performance of the controller proposed in this paper was tested with success, by several simulations, on a bank of realistic simulated patients simulated by means of PK/PD Wiener models obtained from real data collected. The simulation study illustrates the performance of the proposed controller under a variety of clinical conditions, like presence of noise measurement, changing of the doses proportions and of the reference profile.

\section{References}

[1] L. Farina, S. Rinaldi, Positive Linear Systems: Theory and Applications., John Wiley \& Sons, 2000.

[2] W. M. Haddad, T. Hayakawa, J. M. Bailey, International journal of adaptative control and signal processing (2003) 17: 209-235 (DOI: 10.1002/acs.737).

[3] B. Roszak, E. J. Davison, Proceedings of Workshop on Recent Advances in Control and Learning LNCIS 371 (2008) 181-193. 
[4] B. Roszak, E. J. Davison, IEEE Transactions on Automatic Control 55(9) (Sept. 2010) 2204-2209.

[5] T. Kaczorek, Positive 1D and 2D systems, Springer, London, 2002.

[6] J. C. Willems, Journal Automatica (Journal of IFAC) 12(5) (Sept. 1976) $519-523$.

[7] K. Soltesz, G. A. Dumont, K. Heusden, T. Hägglund, 51st IEEE Conference on Decision and Control (Dec. 2012).

[8] G. Bastin, A. Provost, in: Proceedings of the 15th International Symposium on Mathematical Theory of Networks and Systems (2002).

[9] G. Dumont, Proceedings of the 8th IFAC Symposium on Biological and Medical Systems (2012) (2012).

[10] M. M. Silva, T. Mendonça, T. Wigren, Proceedings of the American Control Conference (ACC10) (2010) 4379- 4384.

[11] K. Godfrey, Compartmental Models and Their Application, Academic Press, 1983.

[12] J. P. LaSalle, The Stability of Dynamical Systems, SIAM, Bristol, England, 1976.

[13] R. I. Leine, N. Wouw, Stability and Convergence of Mechanical Systems with Unilateral Constraints, Springer-Verlag, German, 2008.

[14] X. Liao, L. Wang, P. Yu, Stability of Dynamical Systems, Elsevier, Netherlands, 2007. 
[15] F. Bullo, Geometric Control of Mechanical Systems, Springer, USA, 2005 .

[16] J. M. Bailey, W. M. Haddad, Control Systems Magazine, IEEE 25 (2005) $35-51$.

[17] T. Mendonça, H. Alonso, M. M. Silva, S. Esteves, M. Seabra, 16th IFAC Symposium on System Identification (2012).

[18] F. N. Nogueira, T. Mendonça, P. Rocha, Proceedings of the 21st Mediterranean Conference on Control and Automation (MED'13) (2013).

[19] B. Marsh, N. Norton, G. N. Kenny, Br. J. Anaesthesia 67 (1991) 41 48.

[20] C. F. Minto, T. W. Schnider, T. D. Egan, E. Youngs, H. J. Lemmens, P. L. Gambus, V. Billard, J. F. Hoke, K. H. Moore, D. J. Hermann, K. T. Muir, J. W. Mandema, S. L. Shafer, Anesthesiology 86 (1997) 10 $-23$.

[21] T. W. Schnider, C. F. Minto, P. L. Gambus, C. Andresen, D. B. Goodale, S. L. Shafer, E. J. Youngs, Anesthesiology 8 (1998) 1170 - 1182.

\section{Appendix A. Database}

This database was courteously provided by Galeno project (http://www2.fc.up.pt/galeno/).

The parameters presented in Table A.2 were identified in Mendonça et al. $[17]$. 
Table A.1: Patient features

\begin{tabular}{lcccc}
\hline & Gender & Age & Height (cm) & Weight (kg) \\
\hline Patient 1 & F & 56 & 160 & 88 \\
Patient 2 & F & 48 & 158 & 52 \\
Patient 3 & F & 51 & 165 & 55 \\
Patient 4 & F & 56 & 160 & 65 \\
Patient 5 & F & 64 & 146 & 60 \\
Patient 6 & F & 59 & 159 & 110 \\
Patient 7 & F & 29 & 163 & 59 \\
Patient 8 & F & 45 & 155 & 58 \\
Patient 9 & F & 51 & 163 & 55 \\
Patient 10 & F & 32 & 172 & 56 \\
Patient 11 & F & 68 & 160 & 64 \\
Patient 12 & F & 50 & 161 & 68 \\
Patient 13 & F & 68 & 158 & 113 \\
Patient 14 & F & 70 & 161 & 78 \\
Patient 15 & F & 73 & 160 & 75 \\
Patient 16 & F & 34 & 162 & 57 \\
Patient 17 & F & 43 & 155 & 62 \\
Patient 18 & F & 66 & 155 & \\
\hline
\end{tabular}


Table A.2: PPM Parameters

\begin{tabular}{lcccc}
\hline & $\alpha$ & $\eta$ & $\gamma$ & $\mu$ \\
\hline Patient 1 & 0.0667 & 0.3989 & 2.0321 & 4.3266 \\
Patient 2 & 0.0874 & 0.0670 & 1.0133 & 4.3845 \\
Patient 3 & 0.0693 & 0.0482 & 2.0196 & 3.3133 \\
Patient 4 & 0.0590 & 0.0425 & 1.8930 & 4.2273 \\
Patient 5 & 0.0489 & 0.1269 & 1.0702 & 3.9505 \\
Patient 6 & 0.0677 & 0.3373 & 2.6169 & 4.3774 \\
Patient 7 & 0.0737 & 0.2793 & 3.7297 & 4.1494 \\
Patient 8 & 0.0860 & 0.0212 & 0.9172 & 1.0000 \\
Patient 9 & 0.0701 & 0.2837 & 1.8645 & 3.8367 \\
Patient 10 & 0.1041 & 0.1038 & 1.4517 & 3.7978 \\
Patient 11 & 0.0343 & 3.5768 & 0.9334 & 4.4496 \\
Patient 12 & 0.0467 & 0.1254 & 1.6649 & 4.2860 \\
Patient 13 & 0.0687 & 4.5413 & 0.9882 & 3.8094 \\
Patient 14 & 0.0774 & 0.0397 & 3.8213 & 3.2302 \\
Patient 15 & 0.0995 & 0.0377 & 1.6771 & 3.4726 \\
Patient 16 & 0.0929 & 0.1205 & 3.9302 & 3.9983 \\
Patient 17 & 0.0811 & 0.1033 & 1.6096 & 4.2064 \\
Patient 18 & 0.1336 & 0.2307 & 1.5613 & 4.2411 \\
\hline
\end{tabular}

